\title{
Numbers of points of hypersurfaces without lines over finite fields *
}

\author{
Masaaki Homma ${ }^{\dagger}$ \\ Department of Mathematics and Physics \\ Kanagawa University \\ Hiratsuka 259-1293, Japan \\ homma@kanagawa-u.ac.jp
}

\begin{abstract}
We give an upper bound for the number of points of a hypersurface over a finite field that has no lines on, in terms of the dimension, the degree, and the number of the elements of the finite field.

Key Words: Hypersurface, Finite field, Number of points $M S C: 14 \mathrm{~J} 70,14 \mathrm{G} 15,14 \mathrm{G} 05$
\end{abstract}

\section{Introduction}

A few years ago, we proved the Sziklai bound for plane curves in [4, 5, 6]. Let $C$ be a plane curve of degree $d$ over a finite field $\mathbb{F}_{q}$. If $C$ has no $\mathbb{F}_{q}$-line as a component, then the number of $\mathbb{F}_{q}$-points $N_{q}(C)$ of $C$ is bounded by

$$
N_{q}(C) \leq(d-1) q+1
$$

except for the case $d=q=4$ and $C$ is projectively isomorphic over $\mathbb{F}_{4}$ to

$$
K:(X+Y+Z)^{4}+(X Y+Y Z+Z X)^{2}+X Y Z(X+Y+Z)=0 .
$$

This bound is not bad. Actually, for $d=2, \sqrt{q}+1$ (if $q$ is square), $q-$ $1, q, q+1, q+2$, there are nonsingular curves of degree $d$ over $\mathbb{F}_{q}$ that attain the upper bound.

We extended this bound for curves in higher dimensional projective spaces [3]. In this paper, we attempt another generalization of this bound.

We use the notation $\theta_{q}(s)=\frac{q^{s+1}-1}{q-1}$ for any $s \in \mathbb{Z}$. If $s>0, \theta_{q}(s)$ is exactly the number of $\mathbb{F}_{q}$-points of $\mathbb{P}^{s}$. For $s \leq 0, \theta_{q}(0)=1, \theta_{q}(-1)=0$, $\theta_{q}(-2)=-\frac{1}{q}$, etc. The identity $\theta_{q}(s)=q^{s}+\theta_{q}(s-1)$ holds for any $s \in \mathbb{Z}$.

\footnotetext{
* Submitted to to the Fq11 proceedings.

${ }^{\dagger}$ Partially supported by Grant-in-Aid for Scientific Research (24540056), JSPS.
} 
Theorem 1.1 Suppose $n \geq 2$. Let $X$ be a hypersurface in $\mathbb{P}^{n}$ defined over $\mathbb{F}_{q}$ of degree $d$. If $X$ does not contain any $\mathbb{F}_{q}$-lines, then

$$
N_{q}(X) \leq(d-1)\left(q^{n-1}+1\right)+(d-2)\left(\theta_{q}(n-3)-1\right)
$$

except for the case $n=2, d=q=4$ and the curve is projectively isomorphic over $\mathbb{F}_{4}$ to $K$ above.

When $n=2$, this theorem agrees with [6, Theorem 3.1], and several plane curves achieve the upper bound in (2) as mentioned above.

When $n=3$, an elliptic quadric surface has no $\mathbb{F}_{q}$-line and attains the upper bound in (2). As for details, see [1, Chapter 5] and/or [2, Chapter $15]$.

Notation 1.2 For a variety $X$ over $\mathbb{F}_{q}, X\left(\mathbb{F}_{q}\right)$ denotes the set of $\mathbb{F}_{q}$-points of $X$. In particular, $\mathbb{P}^{n}\left(\mathbb{F}_{q}\right)$ is the $n$-dimensional finite projective space over $\mathbb{F}_{q}$. The number of $X\left(\mathbb{F}_{q}\right)$ is denoted by $N_{q}(X)$. $\check{\mathbb{P}}^{n}\left(\mathbb{F}_{q}\right)$ denotes the set of hyperplanes of $\mathbb{P}^{n}$ defined over $\mathbb{F}_{q}$. If $Y$ is a variety defined over a finite extension of $\mathbb{F}_{q}$, the image of $Y$ under the $q$-Frobenius map is denoted by $Y^{(q)}$.

The number of elements of a finite set $S$ is denoted by ${ }^{\#} S$.

\section{The first step}

We prove Theorem 1.1 by induction on $n$. Let $X$ be a hypersurface over $\mathbb{F}_{q}$ of degree $d$ in $\mathbb{P}^{n}$. Let $X=\bigcup_{i} X_{i}$ be the decomposition into $\mathbb{F}_{q}$-irreducible components, and $\operatorname{deg} X_{i}=d_{i}$. If

$$
N_{q}\left(X_{i}\right) \leq\left(d_{i}-1\right)\left(q^{n-1}+1\right)+\left(d_{i}-2\right)\left(\theta_{q}(n-3)-1\right)
$$

holds for each $X_{i}$, we have upper bound (2) for $X$, because $d=\sum_{i} d_{i}$ and $N_{q}(X) \leq \sum_{i} N_{q}\left(X_{i}\right)$. So we assume, a priori, that $X$ is irreducible over $\mathbb{F}_{q}$.

Under the above circumstance, the following lemma holds.

Lemma 2.1 If, for any $H \in \check{\mathbb{P}}^{n}\left(\mathbb{F}_{q}\right)$,

$$
N_{q}(X \cap H) \leq(d-1)\left(q^{n-2}+1\right)+(d-2)\left(\theta_{q}(n-4)-1\right)
$$

holds, then

$$
N_{q}(X) \leq(d-1)\left(q^{n-1}+1\right)+(d-2)\left(\theta_{q}(n-3)-1\right) .
$$

To show the above, the following lemma is needed. 
Lemma 2.2 Let $S$ be a subset of $\mathbb{P}^{n}\left(\mathbb{F}_{q}\right)$. If $\#(S \cap H) \leq \delta$ for any $H \in$ $\check{\mathbb{P}}^{n}\left(\mathbb{F}_{q}\right)$, then

$$
{ }^{\#} S \leq(\delta-1) q+1+\left\lfloor\frac{\delta-1}{\theta_{q}(n-2)}\right\rfloor,
$$

where $\left\lfloor\frac{\delta-1}{\theta_{q}(n-2)}\right\rfloor$ is the integer part of $\frac{\delta-1}{\theta_{q}(n-2)}$.

Proof. See [3, Proposition 2.2].

Proof. [Proof of Lemma 2.1] Let

$$
\delta=(d-1)\left(q^{n-2}+1\right)+(d-2)\left(\theta_{q}(n-4)-1\right) .
$$

Then, from the assumption on $N_{q}(X \cap H)$ and Lemma 2.2.

$$
N_{q}(X) \leq(\delta-1) q+1+\left\lfloor\frac{\delta-1}{\theta_{q}(n-2)}\right\rfloor .
$$

Since

$$
\begin{aligned}
\delta-1 & =(d-2)\left(q^{n-2}+\theta_{q}(n-4)\right)+q^{n-2} \\
& \leq(d-2) \theta_{q}(n-2)+q^{n-2},
\end{aligned}
$$

we have

$$
\frac{\delta-1}{\theta_{q}(n-2)} \leq(d-2)+\frac{q^{n-2}}{\theta_{q}(n-2)} .
$$

Since $q^{n-2}<\theta_{q}(n-2),\left\lfloor\frac{\delta-1}{\theta_{q}(n-2)}\right\rfloor=d-2$. Hence

$$
\begin{aligned}
(\delta-1) q & +1+\left\lfloor\frac{\delta-1}{\theta_{q}(n-2)}\right\rfloor \\
& =\left((d-1)\left(q^{n-2}+1\right)+(d-2)\left(\theta_{q}(n-4)-1\right)-1\right) q+1+d-2 \\
& =\left((d-1) q^{n-2}+(d-2) \theta_{q}(n-4)\right) q+d-1 \\
& =(d-1)\left(q^{n-1}+1\right)+(d-2)\left(\theta_{q}(n-3)-1\right) .
\end{aligned}
$$

This completes the proof.

In the next section, we will show the following theorem.

Theorem 2.3 For an irreducible surface $S$ over $\mathbb{F}_{4}$ of degree 4 in $\mathbb{P}^{3}$, the bound (2) is valid.

Here we complete the proof of Theorem 1.1 under Theorem 2.3 , Proof. [Proof of Theorem 1.1] When $n=2$, the statement of the theorem is the same as [6, Theorem 3.1]. Let us consider the case $n=3$, that is, $X$ is a surface of degree $d$ in $\mathbb{P}^{3}$ which is irreducible over $\mathbb{F}_{q}$. Then we can apply Lemma 2.1 for $X$ except the case $d=q=4$. This exceptional case is just the case where we handle in Theorem 2.3. Therefore the induction on $n \geq 3$ works well by Lemma 2.1. 


\section{$3 \quad$ Surface over $\mathbb{F}_{4}$ of degree 4 in $\mathbb{P}^{3}$}

The aim of this section is to prove Theorem 2.3. An explicit statement is as follows.

Theorem 3.1 Let $S$ be an irreducible surface over $\mathbb{F}_{4}$ of degree 4 in $\mathbb{P}^{3}$, then $N_{4}(S) \leq 51$.

If any $\mathbb{F}_{4}$-plane section $S \cap H$ of $S$ is not $\mathbb{F}_{4}$-isomorphic to the curve $K \subset H=\mathbb{P}^{2}$, one can apply Lemma $2.1(2)$, and get $N_{4}(S) \leq 51$.

We need a property of the plane curve $K$.

Remark 3.2 If a plane curve over $\mathbb{F}_{4}$ of degree 4 is projectively isomorphic to $K$ over $\mathbb{F}_{4}$, then any $\mathbb{F}_{4}$-line of the plane meets the curve at least one $\mathbb{F}_{4}$-point. Indeed, $K\left(\mathbb{F}_{4}\right)=\mathbb{P}^{2}\left(\mathbb{F}_{4}\right) \backslash \mathbb{P}^{2}\left(\mathbb{F}_{2}\right)$ (see [4, Section 3]).

The next lemma is trivial, but meaningful for the proof of Theorem 3.1

Lemma 3.3 Let $Y$ be a surface in $\mathbb{P}^{3}$ over an algebraically closed field. Let $P \in Y$ and $H$ be a plane which is not a component of $Y$ such that $H \ni P$.

(1) Suppose that $P$ is a nonsingular point of $Y$. Then $P$ is a singular point of $Y \cap H$ if and only if $H=T_{P} Y$, where $T_{P} Y$ is the embedded tangent plane to $Y$ at $P$.

(2) If $P$ is a nonsingular point of $Y \cap H$, then it is also a nonsingular point of $Y$.

Now we return to our surface $S$.

Lemma 3.4 If there is a singular $\mathbb{F}_{4}$-point on $S$, then $N_{4}(S)<51$.

Proof. Let $P \in S$ be a singular $\mathbb{F}_{4}$-point, and $\mathcal{L}_{P}$ the set of $\mathbb{F}_{4}$-lines passing through $P$. Then

$$
N_{4}(S)=\sum_{l \in \mathcal{L}_{P}} \#\left(S\left(\mathbb{F}_{4}\right) \cap l \backslash\{P\}\right)+1 .
$$

Since the intersection multiplicity $i(S . l ; P)$ of $S$ and $l$ at $P$ is at least 2 , we have $\#\left(S\left(\mathbb{F}_{4}\right) \cap l \backslash\{P\}\right) \leq 2$. Hence $N_{4}(S) \leq 2 \theta_{4}(2)+1=43$.

By this lemma, we can assume additionally that each point of $S\left(\mathbb{F}_{4}\right)$ is a nonsingular point of $S$.

Lemma 3.5 Let $S$ be an irreducible surface over $\mathbb{F}_{4}$ of degree 4 in $\mathbb{P}^{3}$. Suppose that each $\mathbb{F}_{4}$-point of $S$ is nonsingular. Let $H$ be an $\mathbb{F}_{4}$-plane of $\mathbb{P}^{3}$, and $t(H)={ }^{\#}\left\{P \in S\left(\mathbb{F}_{4}\right) \mid H=T_{P} S\right\}$. Then

(1) $0 \leq t(H) \leq 5$; 
(2) (i) if $t(H)=0$, then ${ }^{\#}\left(S \cap H\left(\mathbb{F}_{4}\right)\right) \leq 14$;

(ii) if $t(H)=1$, then $\#\left(S \cap H\left(\mathbb{F}_{4}\right)\right) \leq 11$;

(iii) if $t(H)=2$, then $\#\left(S \cap H\left(\mathbb{F}_{4}\right)\right) \leq 10$;

(iv) if $t(H)=3$, then $\#\left(S \cap H\left(\mathbb{F}_{4}\right)\right) \leq 8$;

(v) if $t(H)=4$, then $\#\left(S \cap H\left(\mathbb{F}_{4}\right)\right) \leq 6$;

(vi) if $t(H)=5$, then $\#\left(S \cap H\left(\mathbb{F}_{4}\right)\right)=5$.

(3) $t(H)=5$ if and only if $S \cap H$ is a double conic and irreducible as a topological space.

Proof. Since $\operatorname{deg} S \cap H=4, N_{4}(S \cap H) \leq 14$ and equality holds if and only if $S \cap H$ is projectively isomorphic to $K$ over $\mathbb{F}_{4}$ by [6, Theorem 3.1]. From now on, we assume that $t(H)>0$. By Lemma 3.3 and the assumption that each point of $S\left(\mathbb{F}_{4}\right)$ is nonsingular, $t(H)$ coincides with the number of singular $\mathbb{F}_{4}$-points of $S \cap H$.

(Case I) Suppose that $S \cap H$ is absolutely irreducible. Since $S \cap H$ is of degree 4 in $H=\mathbb{P}^{2}$, the arithmetic genus of $S \cap H$ is 3 . Hence the number of singular points is at most 3 . Hence $t(H) \leq 3$.

- When $t(H)=1$, let $P_{0} \in S \cap H\left(\mathbb{F}_{4}\right)$ be the singular point of the curve $S \cap H$. Then, since $i\left(l . S \cap H ; P_{0}\right) \geq 2$ for any $\mathbb{F}_{4}$-line $l$ on $H$,

$$
\begin{aligned}
\#\left(S \cap H\left(\mathbb{F}_{4}\right)\right) & =\sum_{l \in \mathcal{L}_{P_{0}} \cap \check{H}} \#\left(l \cap\left(S \cap H\left(\mathbb{F}_{4}\right)\right) \backslash\left\{P_{0}\right\}\right)+1 \\
& \leq 5 \cdot 2+1=11,
\end{aligned}
$$

where $\mathcal{L}_{P_{0}} \cap \check{H}$ is the set of $\mathbb{F}_{4}$-lines of $H=\mathbb{P}^{2}$ passing through $P_{0}$, which consists of 5 lines.

- When $t(H)=2$, the $\mathbb{F}_{4}$-line passing through two singular points does not meet other points of $\left(S \cap H\left(\mathbb{F}_{4}\right)\right)$. So we have $\#\left(S \cap H\left(\mathbb{F}_{4}\right)\right) \leq 10$ by similar arguments to the above.

- When $t(H)=3$, the normalization of $S \cap H$ at those three points is $\mathbb{P}^{1}$ defined over $\mathbb{F}_{4}$. Hence ${ }^{\#}\left(S \cap H\left(\mathbb{F}_{4}\right)\right) \leq N_{4}\left(\mathbb{P}^{1}\right)+3=8$.

(Case II) Suppose that $S \cap H$ is not absolutely irreducible, but irreducible over $\mathbb{F}_{4}$, which is divided into two sub-cases.

(II-1) Let $S \cap H$ be a union of two absolutely irreducible conics that are conjugate over $\mathbb{F}_{4}$ each other. Then $S \cap H\left(\mathbb{F}_{4}\right)$ is contained in the intersection of those two conics. Hence $t(H) \leq \#\left(S \cap H\left(\mathbb{F}_{4}\right)\right) \leq 4$.

(II-2) Let $S \cap H=l \cup l^{(4)} \cup l^{\left(4^{2}\right)} \cup l^{\left(4^{3}\right)}$, where $l$ is a line over $\mathbb{F}_{4^{4}}$ and not defined over a smaller field. Then $S \cap H\left(\mathbb{F}_{4}\right) \subset l \cap l^{(4)} \cap l^{\left(4^{2}\right)} \cap l^{\left(4^{3}\right)}$. Hence $t(H) \leq \#\left(S \cap H\left(\mathbb{F}_{4}\right)\right) \leq 1$. 
(Case III) Suppose that $S \cap H$ is not irreducible over $\mathbb{F}_{4}$. Since $S \cap H$ has no $\mathbb{F}_{4}$-line as a component, $S \cap H=C_{1} \cup C_{2}$, where $C_{i}$ is a plane curve of degree 2 which is irreducible over $\mathbb{F}_{4}$.

(III-1) If $C_{1}=l \cup l^{(4)}$ and $C_{2}=l^{\prime} \cup l^{\prime(4)}$ for lines $l$ and $l^{\prime}$ over $\mathbb{F}_{4^{2}}$ that are not defined over $\mathbb{F}_{4}$, then $S \cap H\left(\mathbb{F}_{4}\right) \subset\left(l \cap l^{(4)}\right) \cup\left(l^{\prime} \cap l^{\prime(4)}\right)$. Hence $t(H) \leq \#\left(S \cap H\left(\mathbb{F}_{4}\right)\right) \leq 2$.

(III-2) If $C_{1}=l \cup l^{(4)}$ and $C_{2}$ is absolutely irreducible, then $S \cap H\left(\mathbb{F}_{4}\right) \subset$ $\left(l \cap l^{(4)}\right) \cup C_{2}\left(\mathbb{F}_{4}\right)$. Hence $t(H)=1$, and

$$
\#\left(S \cap H\left(\mathbb{F}_{4}\right)\right)=\left\{\begin{array}{ccc}
6 & \text { if } & \left(l \cap l^{(4)}\right) \notin C_{2} \\
5 & \text { if } \quad\left(l \cap l^{(4)}\right) \in C_{2} .
\end{array}\right.
$$

(III-3) If both $C_{1}$ and $C_{2}$ are absolutely irreducible, then we have the following list according the number of $C_{1} \cap C_{2}\left(\mathbb{F}_{4}\right)$. In this case, the set of singular points in $S \cap H\left(\mathbb{F}_{4}\right)$ is just $C_{1} \cap C_{2}\left(\mathbb{F}_{4}\right)$.

\begin{tabular}{ccc}
$\#\left(C_{1} \cap C_{2}\left(\mathbb{F}_{4}\right)\right)$ & $t(H)$ & $\#\left(S \cap H\left(\mathbb{F}_{4}\right)\right)$ \\
\hline 1 & 1 & 9 \\
2 & 2 & 8 \\
3 & 3 & 7 \\
4 & 4 & 6 \\
$C_{1}=C_{2}$ & 5 & 5
\end{tabular}

From the above observations, we have all assertions.

Proof. [Proof of Theorem 3.1] Let $N=N_{4}(S)$ and

$$
n_{i}=\#\left\{H \in \check{\mathbb{P}}^{3}\left(\mathbb{F}_{4}\right) \mid t(H)=i\right\} .
$$

Note that $n_{i}=0$ if $i>5$ by Lemma 3.5 (1). Hence

$$
n_{0}=\theta_{4}(3)-\left(n_{1}+n_{2}+n_{3}+n_{4}+n_{5}\right) .
$$

Recall that each point of $S\left(\mathbb{F}_{4}\right)$ is nonsingular. Hence

$$
n_{1}+2 n_{2}+3 n_{3}+4 n_{4}+5 n_{5}=N \text {. }
$$

First we show that $n_{5}$ can be assumed at most 1 . Let $H_{1}$ and $H_{2}$ be $\mathbb{F}_{4}$-planes in $\mathbb{P}^{3}$ such that $t\left(H_{1}\right)=t\left(H_{2}\right)=5$. Suppose $H_{1} \cap H_{2} \cap S\left(\mathbb{F}_{4}\right) \neq \emptyset$. Choose a point $P \in H_{1} \cap H_{2} \cap S\left(\mathbb{F}_{4}\right)$. For $i=1$ and 2 , since $H_{i} \cap S$ is a double conic, $P$ is a singular point of $H_{i} \cap S$. Hence $H_{i}=T_{P}(S)$ by Lemma 3.3, and hence $H_{1}=H_{2}$. Suppose the contrary: $H_{1} \cap H_{2} \cap S\left(\mathbb{F}_{4}\right)=\emptyset$ and $H_{1} \neq H_{2}$. Let $l$ be the $\mathbb{F}_{4}$-line $H_{1} \cap H_{2}$, and $H_{3}^{\prime}, H_{4}^{\prime}, H_{5}^{\prime}$ the other three $\mathbb{F}_{4}$-planes containing the line $l$. Then, for $j=3,4$ and $5, H_{j}^{\prime} \cap S$ is not $\mathbb{F}_{4}$-isomorphic to $K$. In fact, since $l$ is an $\mathbb{F}_{4}$-line on the plane $H_{j}^{\prime}$ and 
$l \cap\left(H_{j}^{\prime} \cap S\left(\mathbb{F}_{4}\right)\right) \subset l \cap S\left(\mathbb{F}_{4}\right)=\emptyset, H_{j}^{\prime} \cap S$ cannot be projectively isomorphic to $K$ over $\mathbb{F}_{4}$ by Remark 3.2. Hence $N_{4}\left(H_{j}^{\prime} \cap S\right) \leq 13$. Therefore

$$
\begin{aligned}
N_{4}(S) & =N_{4}\left(H_{1} \cap S\right)+N_{4}\left(H_{2} \cap S\right)+\sum_{j=3}^{5} N_{4}\left(H_{j}^{\prime} \cap S\right) \\
& \leq 5+5+3 \times 13=49,
\end{aligned}
$$

which means that the target inequality already holds in this case.

Consider the correspondence

$$
\mathcal{P}=\left\{(P, H) \in S\left(\mathbb{F}_{4}\right) \times \check{\mathbb{P}}^{3}\left(\mathbb{F}_{4}\right) \mid P \in H\right\}
$$

with two projections $\pi_{1}: \mathcal{P} \rightarrow S\left(\mathbb{F}_{4}\right)$ and $\pi_{2}: \mathcal{P} \rightarrow \check{\mathbb{P}}^{3}\left(\mathbb{F}_{4}\right)$. By using $\pi_{1}$, we have ${ }^{\#} \mathcal{P}=N \theta_{4}(2)$. On the other hand, by using $\pi_{2}$,

$$
\begin{aligned}
\#_{\mathcal{P}} & =\sum_{j=0}^{5} \sum_{\substack{H \text { with } \\
t(H)=j}} \#\left(H \cap S\left(\mathbb{F}_{4}\right)\right) \\
& \leq 14\left(\theta_{4}(3)-\left(n_{1}+\cdots+n_{5}\right)\right)+11 n_{1}+10 n_{2}+8 n_{3}+6 n_{4}+5 n_{5}
\end{aligned}
$$

(by Lemma 3.5)

$$
\begin{aligned}
& =14 \theta_{4}(3)-2\left(\sum_{j=1}^{5} j n_{j}\right)+n_{5}-n_{1} \\
& =14 \theta_{4}(3)-2 N+n_{5}-n_{1} \\
& \left.\leq 14 \theta_{4}(3)-2 N+1 \text { (because } n_{5} \leq 1\right) .
\end{aligned}
$$

Therefore

$$
N\left(\theta_{4}(2)+2\right) \leq 14 \theta_{4}(3)+1=1191,
$$

and then $N \leq\left\lfloor 51+\frac{18}{23}\right\rfloor=51$. This completes the proof.

\section{References}

[1] J. W. P. Hirschfeld, Projective geometries over finite fields, Oxford University Press, Oxford, 1979.

[2] J. W. P. Hirschfeld, Finite projective spaces of three dimensions, Clarendon Press, Oxford, 1985.

[3] M. Homma, A bound on the number of points of a curve in a projective space over a finite field, in: M. Lavrauw, G.L. Mullen, S. Nikova, D. Panario, and L. Storme (Eds.), Theory and Applications of Finite Fields, 103-110 Contemp. Math., vol. 579, AMS, Providence, 2012. 
[4] M. Homma and S. J. Kim, Around Sziklai's conjecture on the number of points of a plane curve over a finite field, Finite Fields and Their Applications 15 (2009), 468-474.

[5] M. Homma and S. J. Kim, Sziklai's conjecture on the number of points of a plane curve over a finite field II, in: G. McGuire, G.L. Mullen, D. Panario, I.E. Shparlinski (Eds.), Finite Fields: Theory and Applications, in: Contemp. Math., vol. 518, AMS, Providence, 2010, 225-234. (An update is available at arXiv 0907.1325v2.)

[6] M. Homma and S. J. Kim, Sziklai's conjecture on the number of points of a plane curve over a finite field III, Finite Fields Appl. 16 (2010) 315-319. 